\title{
Universality and backreaction in a general-relativistic accretion of steady fluids
}

\author{
Janusz Karkowski, ${ }^{1}$ Bogusz Kinasiewicz, ${ }^{1}$ Patryk Mach, ${ }^{1}$ Edward Malec, ${ }^{1,2,3}$ and Zdobysław Świerczyński ${ }^{4}$ \\ ${ }^{1}$ M. Smoluchowski Institute of Physics, Jagiellonian University, Reymonta 4, 30-059 Kraków, Poland \\ ${ }^{2}$ AEI MPI, Golm, Germany \\ ${ }^{3}$ TPI FSU, Jena, Germany \\ ${ }^{4}$ Pedagogical University, Podchorazżych 1, Kraków, Poland
}

\begin{abstract}
The spherically symmetric steady accretion of polytropic perfect fluids onto a black hole is the simplest flow model that can demonstrate the effects of backreaction. The analytic and numerical investigation reveals that backreaction keeps intact most of the characteristics of the sonic point. For any such system, with the free parameter being the relative abundance of the fluid, the mass accretion rate achieves maximal value when the mass of the fluid is $1 / 3$ of the total mass. Fixing the total mass of the system, one observes the existence of two weakly accreting regimes, one over-abundant and the other poor in fluid content.
\end{abstract}

The spherical steady accretion of gas onto a gravitational center has been investigated in the Newtonian context by Bondi [1] and in the Schwarzschild space-time by Michel [2], Shapiro and Teukolsky [3], and others [4]. The Newtonian critical accretion flow was shown to be stable by Balazs and others [5], while the stability of the steady flow in the fixed Schwarzschild background has been analyzed by Moncrief [6]. The general-relativistic description including backreaction has been formulated in [8]. There are two reasons for inspecting steady flows in the full generality, when infalling gas modifies the space-time geometry, that is including backreaction [7]. First, one can see the effects of backreaction in a simple but nontrivial accretion model. Second, gravitational collapse of the fluid which starts as a flow dominated by a steady accretion, can be better controlled, and that would allow one to have an insight into formation of gravitational singularities. This paper focuses on the issue of backreaction. We show that the backreaction strongly influences the mass accretion rate.

We will consider a spherically symmetric compact ball of a fluid falling onto a non-rotating black hole. The black hole provides the simplest choice for the central object since one can neglect the occurrence of shock waves. The fluid is regarded to be steady in a sense defined later and by a black hole we understand the existence of an apparent horizon. For the detailed derivation of equations the reader should consult [8]. Here we give only a brief description, focusing attention on the physical assumptions. We will use comoving coordinates

$$
d s^{2}=-N^{2} d t^{2}+\alpha d r^{2}+R^{2}\left(d \theta^{2}+\sin ^{2} \theta d \phi^{2}\right)
$$

where the lapse $N, \alpha$ and the areal radius $R$ are functions of a coordinate radius $r$ and an asymptotic time variable $t$. The nonzero components of the extrinsic curvature $K_{i j}$ of the $t=$ const slices $\operatorname{read} K_{r}^{r}=\frac{1}{2 N \alpha} \partial_{t} \alpha, \quad K_{\phi}^{\phi}=K_{\theta}^{\theta}=\frac{\partial_{t} R}{N R}=$ $\frac{1}{2}\left(\operatorname{tr} K-K_{r}^{r}\right)$. Here $\operatorname{tr} K=\frac{1}{N} \partial_{t} \ln \left(\sqrt{\alpha} R^{2}\right)$. The mean curvature of two-spheres of constant radius $r$, embedded in a Cauchy hypersurface is $p=\frac{2 \partial_{r} R}{R \sqrt{\alpha}}$. The energy-momentum tensor of the perfect fluid reads $T^{\mu \nu}=(\tilde{p}+\varrho) u^{\mu} u^{v}+\tilde{p} g^{\mu \nu},\left(u_{\mu} u^{\mu}=-1\right)$ where $u^{\mu}$ denotes the four-velocity of the fluid, $\tilde{p}$ is the pressure and $\varrho$ the energy density in the comoving frame.
The areal velocity $U$ of a comoving particle designated by coordinates $(r, t)$ is given by $U(r, t)=\frac{\partial_{t} R}{N}=\frac{R}{2}\left(\operatorname{tr} K-K_{r}^{r}\right)$. From the Einstein constraint equations one has

$$
p R=2 \sqrt{1-\frac{2 m(R)}{R}+U^{2}}
$$

and $\partial_{R}\left(R^{2} U\right)-R^{2} \operatorname{tr} K=0$. We use here and in what follows the relation $\partial_{r}=\sqrt{\alpha}(p R / 2) \partial_{R}$ in order to eliminate the differentiation with respect the comoving radius $r$. The quasilocal mass $m(R)$ obeys the equation $\partial_{R} m(R)=4 \pi R^{2} \varrho$. The mass accretion rate is $\dot{m}=\left(\partial_{t}-\left(\partial_{t} R\right) \partial_{R}\right) m(R)=-4 \pi N R^{2} U(\varrho+\tilde{p})$. A more familiar form of that is $\dot{m}=-4 \pi R^{2} n U$, where $n$ is the baryonic density (see below). A standard condition for the steady collapsing fluid is that all its characteristics are constant at a fixed $R$ (see [9]). In analytical terms $\left.\partial_{t} X\right|_{R=\text { const }}=$ $\left(\partial_{t}-\left(\partial_{t} R\right) \partial_{R}\right) X=0$, where $X=\varrho, U, a \ldots[10]$. The Einstein evolution equation $\partial_{t} U=p^{2} R^{2} \partial_{R} N / 4-m(R) N / R^{2}-4 \pi N R \tilde{p}$ and the energy conservation equation $\partial_{t} \varrho=-N \operatorname{tr} K(\varrho+\tilde{p})$ become ordinary differential equations with respect $R$, assuming steady flow. Selfgravitating steady fluids still allow for the change in time of some geometric quantities, like the mean curvature $p$ or the area of the black hole. It is easy to show that for a steady flow $\partial_{R} \dot{m}=0$ [8].

By a black hole is meant a region within an apparent horizon to the future, i.e., a region enclosed by an outermost sphere $S_{A}$ on which the optical scalar $\theta_{+} \equiv \frac{p R}{2}+U$ vanishes [12]. (The other condition, that $\theta_{-} \equiv \frac{p R}{2}-U>0$ for all spheres outside $S_{A}$, is satisfied trivially for a steady accretion.) That means that on $S_{A}$ the ratio $2 m_{B H} / R_{A H}$ becomes 1 , where $R_{A H}$ is the areal radius of the apparent horizon and $m_{B H} \equiv m\left(R_{A H}\right)$ is the mass of the black hole. We shall specialize to polytropic perfect fluids $\tilde{p}=K \varrho^{\Gamma}$, with $\Gamma$ being a constant, $(1 \leq \Gamma \leq 5 / 3)$.

Furthermore, assume the radius $R_{\infty}$ of the ball of fluid and boundary data are such that $\left|U_{\infty}\right| \ll \frac{m\left(R_{\infty}\right)}{R_{\infty}} \ll a_{\infty}$. These boundary conditions are needed in order to glue the steady fluid with the external Schwarzschild geometry (see a discussion later). The momentum conservation equation $\nabla_{\mu} T_{r}^{\mu}=0$ — in comoving coordinates

$$
N \partial_{R} \tilde{p}+(\tilde{p}+\varrho) \partial_{R} N=0
$$


— can be integrated, yielding (with $N\left(R_{\infty}\right)=1$ )

$$
a^{2}=-\Gamma+\frac{\Gamma+a_{\infty}^{2}}{N^{\kappa}} .
$$

Here $\kappa=\frac{\Gamma-1}{\Gamma}$. (4) can be regarded as the general-relativistic version of the Bernoulli equation. Another useful relation that follows from (3) is $N=\frac{C n}{\tilde{p}+\varrho}$. Here appears the baryonic number density $n=C \exp \int_{\varrho_{\infty}}^{\varrho} d s \frac{1}{s+\tilde{p}(s)}$. The baryonic number density works, for perfect barotropes, as an integration factor; if $T_{\mu \nu}$ is conserved, then $\nabla_{\mu}\left(n u^{\mu}\right)=0$. This representation of the lapse function shows that the lapse $N$ is constant along an orbit of the constant areal radius $R$.

In the general-relativistic case the sonic point is defined as a location where the length of the spatial velocity vector $|\vec{U}|=|U| /(p R / 2)$ equals $a$. Therefore at a sonic point $|U|=\frac{1}{2} p R a$. In the Newtonian limit this coincides with the standard requirement $|U|=a$. In the following we will denote by the asterisk all values referring to the sonic points, e.g. $a_{*}, U_{*}$, etc. The four characteristics, $a_{*}, U_{*}, m_{*} / R_{*}$ and $c_{*}$ are related [8], $a_{*}^{2}\left(1-\frac{3 m_{*}}{2 R_{*}}+c_{*}\right)=U_{*}^{2}=\frac{m_{*}}{2 R_{*}}+c_{*}$, where $c_{*}=2 \pi R_{*}^{2} \tilde{p}_{*}$. The infall velocity $U$ reads

$$
U=U_{*} \frac{R_{*}^{2}}{R^{2}}\left(\frac{1+\frac{\Gamma}{a^{2}}}{1+\frac{\Gamma}{a_{*}^{2}}}\right)^{1 /(\Gamma-1)} .
$$

Here $U_{*}$ is the negative square root. From the relation between the pressure and the energy density, one obtains, using equation (4)

$$
\varrho=\varrho_{\infty}\left(a / a_{\infty}\right)^{2 /(\Gamma-1)}=\varrho_{\infty}\left(-\frac{\Gamma}{a_{\infty}^{2}}+\frac{\frac{\Gamma}{a_{\infty}^{2}}+1}{N^{K}}\right)^{\frac{1}{\Gamma-1}},
$$

where the constant $\varrho_{\infty}$ is equal to the mass density of a collapsing fluid at the boundary $R_{\infty}$. The steady fluid is described by equations (4-6). They constitute an integro-algebraic system of equations, with a bifurcation point at the sonic point, where two branches (identified as accretion or wind) do cross; that is a well known feature of that problem, present also in models with a test fluid [1] - [5], [8]. That requires some caution in doing numerics and a careful selection of the solution branch. Notice that these equations are expressed exclusively in terms of quantities that are steady in the sense of the former definition.

This collapsing compact cloud can be connected to the Schwarzschild exterior. The ideal model of a steady flow would be that engineered by a "daemon" who just fixes boundary data conditions; a similar picture might well be valid in some binaries. Alternatively, one can imagine an initial configuration with a steady fluid annulus around the black hole and an external transition zone that is not steady (since both pressure and the mass density must be made to vanish there) and that can expand outward and inward with the speed of sound, perturbing the external layers of the steady interior. The boundary can be connected at $R_{\infty}$, via an almost massless transition zone, to the external Schwarzschild geometry; the standard junction conditions - continuity of the metric and of the transversal extrinsic curvature, and the condition $N_{\infty}=1$ - require now the appropriate choice of the slicing of the exterior region. We verified in a number of numerical calculations that for short evolution times $\left(t \ll R_{\infty} / a_{\infty}\right)$ and under the former boundary conditions the bulk of the fluid is steady. (Notice, that this observation indicates that such initial data can be useful in order to model the gravitational collapse.) The asymptotic mass $m$ of the spacetime would be well approximated by $m_{\infty}$, the sum of the masses of the black hole and the quasi-stationary gas, for a suitably chosen transition zone.

In numerical calculations it will be convenient to represent the mass $m(R)$ in the form

$$
m(R)=m-4 \pi \int_{R}^{R_{\infty}} d r r^{2} \varrho .
$$

Notice that $m(\infty)=m_{\infty} \approx m$. Assuming steady flow, one can obtain following expression for the lapse $N$ [8]

$$
\begin{aligned}
N & =\frac{p R}{p\left(R_{\infty}\right) R_{\infty}} \beta(R), \\
\beta(R) & =\exp \left(-16 \pi \int_{R}^{R_{\infty}}(\tilde{p}+\varrho) \frac{d s}{p^{2} s}\right) .
\end{aligned}
$$

The full description of the steady accretion that will be discussed later is given by equations (2), (7), (4), (5) and (8); this is equivalent to the former system.

One of two main results of this paper is realization that significant information about the full system with backreaction can be obtained through the investigation of steady flows with the backreaction being ignored.

Namely, let be given $\Gamma \in(1,5 / 3]$, asymptotic data $\varrho_{\infty}$ and $a_{\infty}$ and let $\left|U_{\infty}\right| \ll \frac{m\left(R_{\infty}\right)}{R_{\infty}} \ll a_{\infty}$. Assume that the sonic point is located outside the apparent horizon, i.e., $U_{*}^{2}<0.25$. Then the sonic point parameters $a_{*}^{2}, U_{*}^{2}$ and $m_{*} / R_{*}$ in the above model with backreaction are the same as in the test fluid accretion with the same asymptotic data [11].

Sketch of the reasoning. We will apply the bootstrap-type argument. Let us assume temporarily that $U_{*}^{2}<0.1$, that is $R_{*}>5 m_{*}$. This implies $p R / 2 \geq \sqrt{0.7}, a^{2} \leq a_{*}^{2} \leq 1 / 7$ and $16 \pi \int_{R}^{R_{\infty}}(\tilde{p}+\varrho) \frac{1}{p^{2} s} d s \leq 80 \frac{m-m\left(R_{*}\right)}{49 R_{*}}$ for $R \geq R_{*}$.

If $m-m\left(R_{*}\right) \leq(49 / 80) m\left(R_{*}\right)$, then the integrand present in the exponent of the function $\beta(r)$ is smaller than $m_{*} / R_{*}$. That in turn means that $N \geq \sqrt{1-\frac{4 m_{*}}{R_{*}}+U_{*}^{2}}$ and

$$
a_{*}^{2} \leq-\Gamma+\frac{\Gamma+a_{\infty}^{2}}{{\sqrt{1-\frac{4 m_{*}}{R_{*}}+U_{*}^{2}}}^{k}} .
$$

Solutions of this inequality are bounded from above by the solution of the equation

$$
\tilde{a}^{2}=-\Gamma+\frac{\Gamma+a_{\infty}^{2}}{{\sqrt{1-\frac{2 \tilde{m}}{R_{*}}+\tilde{U}^{2}}}^{\kappa}},
$$


where $\tilde{m}=2 m_{*}$, at the point where $\tilde{a}^{2} /\left(1+3 \tilde{a}^{2}\right)=\tilde{m} /\left(2 R_{*}\right)=$ $\tilde{U}^{2}$. Note that $R_{*}>2 \tilde{m}$; this allows us to use the estimate of Theorem 2 of [8]. Thus one has

$$
a_{*}^{2}<\tilde{a}^{2} \leq \frac{2 a_{\infty}^{2}}{5-3 \Gamma+\frac{3 \tilde{a}^{2}(\Gamma-1)^{2}(9 \Gamma-7)}{4 \Gamma\left(1+3 \tilde{a}^{2}\right)}} .
$$

Inserting this estimate into $[$, one can bound the matter density, $\varrho(R) \leq \varrho_{\infty}\left(1+C\left(\Gamma, a_{*}\right) /\left(5-3 \Gamma+a_{*}^{2}\right)\right)$. Here the constant $C$ is of the order of 10 . This in turn allows one to replace the bound on the exponent of $\beta$ by a much better one of the or$\operatorname{der}\left(m_{*} / R_{*}\right)\left(R_{*} / R_{\infty}\right)$; this is clearly negligible in comparison to $m_{*} / R_{*}$ (save for a small region around $\Gamma=5 / 3$ ) and one can repeat the previous argument, but this time assuming $\beta=1$. This would lead to the improved estimate of $a$ and $\varrho$ outside $R=5 R_{*}$. Further refinement - the extension into bigger values of $U_{*}^{2}$ and of $m_{*} / m$, and to all $\Gamma$ 's in the interval $(1,5 / 3$ ] - would be done by splitting the integral of $\beta$ into two integrals, $\int_{R_{*}}^{R_{\infty}} \ldots=\int_{R_{*}}^{5 R_{*}} \ldots+\int_{5 R_{*}}^{R_{\infty}} \ldots$ and separately estimating each term.

Using the preceding estimates, one gets

$$
c_{*}=\frac{a_{*}^{2}}{\Gamma} 2 \pi R_{*}^{2} \varrho_{*} \leq C a_{*}^{2} \frac{m_{*}}{R_{\infty}} \frac{R_{*}^{2}}{R_{\infty}^{2}} \ll \frac{m_{*}}{R_{*}} .
$$

That implies that both $c_{*}$ and the exponent of $\beta$ can be put to zero in all relations between characteristics of the sonic point. Inserting this information into the Bernoulli equation (4) one obtains that at a sonic point [8]

$$
1+y(3 \Gamma-1)=3\left(a_{\infty}^{2}+\Gamma\right) y^{\frac{\Gamma+1}{2 \Gamma}},
$$

where $y=1-3 m_{*} /\left(2 R_{*}\right)$. Coefficients of this algebraic equation do not depend on the asymptotic mass density and therefore $y$ is $\varrho_{\infty}$-independent. The sonic mass $m_{*}$ and the sonic radius $R_{*}$ clearly depend on $\varrho_{\infty}$ but their ratio is constant. In fact, $m_{*} / R_{*}$ must be exactly the same as in the case when the backreaction can be neglected, that is when the mass of the fluid outside the black hole is small in comparison to the total mass. The same conclusion holds true also for other parameters of the sonic point, the fluid velocity $U_{*}$ and the speed of sound $a_{*}$. In conclusion: $a_{*}^{2}, U_{*}^{2}$ and $m_{*} / R_{*}$ can be inferred from a suitable steady flow with a test fluid.

It follows from the above discussion that the mass density changes moderately outside the sonic point. In fact, for $R \in\left[R_{*}, 10 R_{*}\right] \varrho(R) \leq C(R) \varrho_{\infty}$ with $C\left(R_{*}\right)$ being of the order of 10 and $C\left(10 R_{*}\right)$ being slightly larger than 1 . Assuming that $R_{\infty} \gg 10 R_{*}$ one can show (taking into account the fact, that the region $\left(10 R_{*}, R_{\infty}\right)$ has a much larger volume than $\left.\left(R_{*}, 10 R_{*}\right)\right)$ that under the preceding assumptions the mass of outer layers of the fluid $m-m_{*}=4 \pi \int_{R_{*}}^{R_{\infty}} d r r^{2} \varrho=\gamma \varrho_{\infty}$ with a constant $\gamma$ determined in practice only by $R_{\infty}$. Alternatively, $m_{*} / m=1-\varrho_{\infty}(\gamma / m)$; the ratio $m_{*} / m$ is a linearly decreasing function of $\varrho_{\infty}$.

The rate of the mass accretion $\dot{m}$ within the steadily accret- ing fluid can be expressed as below (see Eq. (6.1) in [8])

$$
\dot{m}=\pi m_{*}^{2} \varrho_{\infty} \frac{R_{*}^{2}}{m_{*}^{2}}\left(\frac{a_{*}^{2}}{a_{\infty}^{2}}\right)^{\frac{(5-3 \Gamma)}{2(\Gamma-1)}}\left(1+\frac{a_{*}^{2}}{\Gamma}\right) \frac{1+3 a_{*}^{2}}{a_{\infty}^{3}} .
$$

The whole dependence on $\varrho_{\infty}$ is contained in the factor $m_{*}^{2} \varrho_{\infty}$. This achieves (fixing $m$ ) a maximum at $m_{*}=2 \mathrm{~m} / 3$ and tends to zero at both ends: i) $m_{*} \rightarrow m$ (when the density $\varrho$ tends to zero) and ii) $m_{*} / m \rightarrow 0$ (when the mass of black hole is negligible in comparison to the mass of the fluid). In this place we invoked the assumptions concerning boundary conditions.

Thus, one arrives at our second main result: Amongst steadily accreting systems of the same $R_{\infty}, a_{\infty}$ and $m$ those will be most efficient for which $m_{*}=2 m / 3$. The factor $2 / 3$ is universal - independent of the parameters $R_{\infty}, \Gamma$ and $a_{\infty}$.

Since the mass of the fluid $m_{f}$ is close to $m-m_{*}$ and $m=$ $m_{f}+m_{B H}$, the above means that the maximum of the mass accretion takes place when the mass of the fluid is a half of the mass of the black hole. We would like to point that in (14) the mass $m_{*}$ is only approximately constant, and hence $\dot{m}$ is not strictly constant in time. The fulfilment of $\dot{m} \tau \ll m_{*}$, where $\tau$ is the evolution time, can be understood as yet another criterion for the validity of the steady flow approximation.

In the remaining part of the paper the asymptotic mass $m$ has been normalized to 1 . In order to test the above results, we performed a series of numerical calculations, according to the following scenario. i) Fix a value of the adiabatic index $\Gamma$ and the asymptotic speed of sound $a_{\infty}^{2}$; change in a systematic way the asymptotic mass density. ii) For the same $\Gamma$ choose new $a_{\infty}^{2}$ and change the asymptotic mass density. iii) Choose new $\Gamma$ and repeat the preceding procedure. Below we shall describe only a small subset of the numerical evidence.

TABLE I: Characteristics of the sonic point $a_{*}^{2},\left|U_{*}\right|, R_{*}$ for the $\Gamma=$ $4 / 3$ polytrope and $a_{\infty}^{2}=0.1$. The last column shows the areal radius of the apparent horizon. The mass accretion rate (second column) achieves maximum at $m_{B H} \approx 0.663$.

\begin{tabular}{cccccc}
$m_{f} / m$ & $\dot{m}$ & $R_{*}$ & $U_{*}$ & $a_{*}^{2}$ & $R_{A H}$ \\
\hline \hline $4.186 \times 10^{-32}$ & $4.156 \times 10^{-48}$ & 4.290 & 0.34137 & 0.179184 & 1.993 \\
$4.186 \times 10^{-17}$ & $4.156 \times 10^{-33}$ & 4.290 & 0.34137 & 0.179184 & 1.993 \\
0.041 & $3.815 \times 10^{-18}$ & 4.110 & 0.341369 & 0.179182 & 1.906 \\
0.083 & $6.978 \times 10^{-18}$ & 3.931 & 0.341371 & 0.179183 & 1.821 \\
0.167 & $1.152 \times 10^{-17}$ & 3.571 & 0.341371 & 0.179182 & 1.656 \\
0.251 & $1.397 \times 10^{-17}$ & 3.212 & 0.341371 & 0.179183 & 1.492 \\
0.333 & $1.469 \times 10^{-17}$ & 2.859 & 0.341371 & 0.179183 & 1.326 \\
0.418 & $1.403 \times 10^{-17}$ & 2.493 & 0.341399 & 0.179184 & 1.156 \\
0.628 & $8.612 \times 10^{-18}$ & 1.594 & 0.341369 & 0.179182 & 0.740 \\
0.837 & $2.187 \times 10^{-18}$ & 0.696 & 0.341394 & 0.179185 & 0.322 \\
0.879 & $1.264 \times 10^{-18}$ & 0.516 & 0.341399 & 0.179184 & 0.239 \\
0.962 & $1.277 \times 10^{-19}$ & 0.156 & 0.34137 & 0.179187 & 0.072 \\
0.983 & $2.382 \times 10^{-20}$ & 0.067 & 0.341399 & 0.179185 & 0.031
\end{tabular}

It appears that the characteristics $a_{*}^{2}, U_{*}^{2}, m_{*} / R_{*}$ of the sonic point practically do not depend, for a given $\Gamma$ and $a_{\infty}^{2}$, on the energy density $\varrho_{\infty}$ (exemplary results are shown in Table 
1 ), suggesting a precision of $10^{-5}$. The more reliable measure of the numerical error is the difference between the mass $m=1$ (assumed in the equations) and the mass found numerically. Our results (see Table 1) suggest that numerical error is smaller than $0.5 \%$. The quantity $c_{*}$ is negligible in comparison to other sonic point parameters. The mass $m_{*}$ behaves like $m_{*}=1-\gamma \varrho_{\infty}$ (Fig. 1). And finally, the extremum of $\dot{m}$ is achieved when $m_{*} \approx 2 / 3$ (Fig. 2). Notably, numerical results extend the regime in which the universality is observed into fluids characterized by the speed of sound $a_{\infty}$ exceeding 1. This is remarkable, since that implies $R_{*}<R_{A H}$ and the theoretical analysis presented above would not be valid.

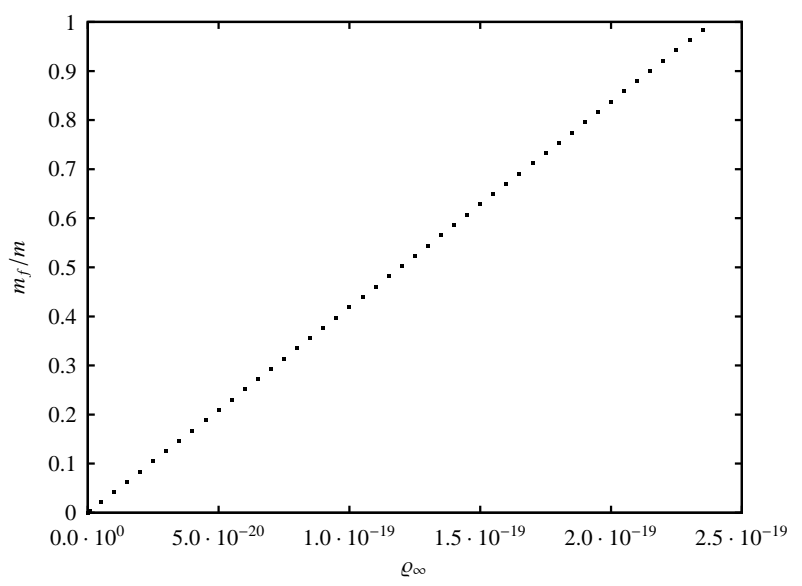

FIG. 1: The dependence of $\frac{m_{f}}{m} \approx 1-m_{*}$ on the asymptotic mass density.

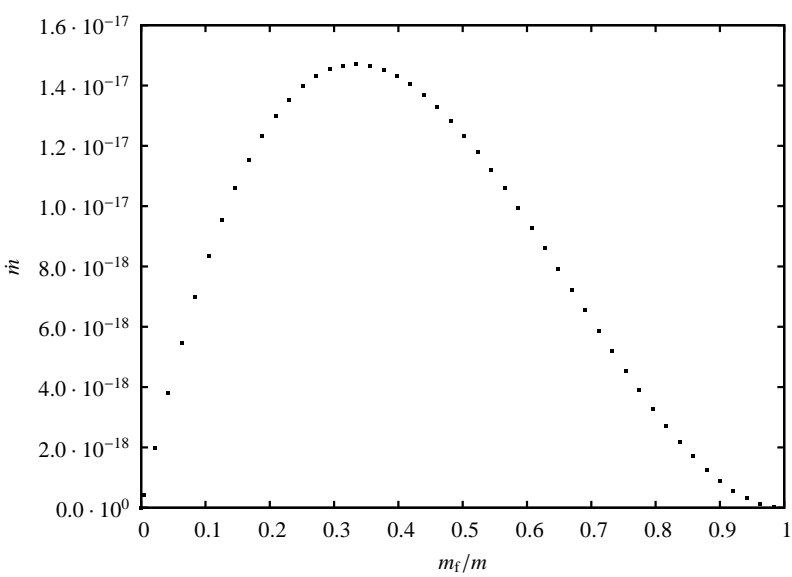

FIG. 2: The dependence of $\dot{m}$ on the ratio $\left(m-m_{*}\right) / m$.

Formula (14) implies that the mass accretion rate in the case with backreaction (when $m_{*}<m$ ) can be only smaller from the mass accretion rate without backreaction (when $m_{*}=m$ ), with the same asymptotic parameters $\rho_{\infty}, a_{\infty}, m$. Numerical results confirm the preceding argument that $\gamma$ depends essentially only on the size of support.

In the case of polytropes with $\tilde{p}=K n^{\Gamma}$ the Bernoulli equa- tion reads $N\left(\Gamma-1-a_{\infty}^{2}\right)=\Gamma-1-a^{2}$. The analytic arguments of the type presented above can be applied and conclusions are the same. Similar results should be true for Newtonian massive fluids for $\Gamma$ significantly smaller than $5 / 3$.

In conclusion, in the simple model of accretion with backreaction considered here, one can get all parameters describing the sonic point, with the exception of its location $R_{*}$ and mass $m_{*}$ simply from a related polytropic model with the test fluid having the same index $\Gamma$ and the same asymptotic speed of sound $a_{\infty}$. The main result is that the mass accretion rate $\dot{m}$ achieves maximum at $m_{f} / m_{B H} \approx 1 / 2$; therefore there exist two different regimes, $m_{f} / m_{B H} \ll 1$ and $m_{f} / m_{B H} \gg 1$, with low accretion. In contrast to that, in the test fluid approximation the quantity $\dot{m}$ grows with $\varrho_{\infty}$ (that is with $m_{f} / m_{B H}$ ). The mass accretion rate $\dot{m}$ sets the upper limit for the luminosity of the system. Hence this simple analysis of accretion suggests the existence of two weakly luminous regimes: one rich in fluid with $m_{f} / m_{B H} \gg 1$ and the other with a small amount of fluid, $m_{f} / m_{B H} \ll 1$. We noticed also that the normalized efficiency of accretion (defined as $\dot{m} / m_{B H}$ ) goes to zero.

Results of this paper show the importance of the backreaction. We believe that the qualitative features demonstrated by the spherically symmetric model - the dependence of the mass accretion on $m_{f} / m_{B H}$ and the existence of two weakly accreting regimes - will appear in the descriptions of accreting systems with angular momentum.

Acknowledgements. EM thanks Bernd Schmidt for discussions. This paper is partially supported by the MNII grant 1PO3B 01229.

[1] H. Bondi, Mon. Not. R. Astron. Soc. 112, 192(1952).

[2] F. C. Michel, Astrophys. Space Sci. 15, 153(1972).

[3] S. Shapiro and S. Teukolsky, Black Holes, White Dwarfs and Neutron Stars, Wiley, New York, 1983.

[4] T.K. Das, Astron. Astrophys. 374, 1150(2001); B. Kinasiewicz and T. Lanczewski, Acta Phys. Pol. B36, 1951(2005).

[5] N. L. Balazs, Mon. Not. R. Astr. Soc. 160, 79(1972); R. F. Stellingwerf and J. Buff, Astrophys. J. 198, 671(1978); A. R. Garlick, Astr. Astrophysics, 73, 171(1979); J. Patterson, J. Silk and J. P. Ostriker, Mon. Not. R. Astr. Soc., 191, 571(1980).

[6] V. Moncrief, Astrophys. J. 235, 1038(1980).

[7] R. Wald, General Relativity, Chicago, University of Chicago Press, 1984.

[8] E. Malec, Phys. Rev. D60, 104043 (1999).

[9] R. Courant and K. O. Friedrichs, Supersonic Flow and Shock Waves, Appl. Math. Sc. 21, Springer-Verlag.

[10] Notice that $\left(\partial_{t}-\left(\partial_{t} R\right) \partial_{R}\right) \propto \partial_{T}$, where $T$ is the Misner-Sharp time, see: C. W. Misner and D. H. Sharp, Phys. Rev. 136B, 571(1964).

[11] In the test fluid approximation the parameters of the sonic point do not depend on the central mass. See Theorem 2 and Eqs. $(5.10-5.11)$ in $[8]$.

[12] E. Malec and N. O'Murchadha, Phys. Rev. D50, 6033(1994). 